\title{
A New Criterion for Bounded Component Analysis
}

This paper was downloaded from TechRxiv (https://www.techrxiv.org).

\section{LICENSE}

CC BY 4.0

SUBMISSION DATE / POSTED DATE

05-10-2021 / 13-10-2021

CITATION

Brotto, Renan; Nose-Filho, Kenji; Romano, João M. T. (2021): A New Criterion for Bounded Component Analysis. TechRxiv. Preprint. https://doi.org/10.36227/techrxiv.16744216.v1

$\mathrm{DOI}$ 


\title{
A New Criterion for Bounded Component Analysis
}

\author{
Renan D. B. Brotto, Kenji Nose-Filho, João M. T. Romano
}

\begin{abstract}
In this paper we present a new criterion for bounded component analysis, a quite new approach for the Blind Source Separation problem. For the determined case, we show that the $\ell_{\infty}$-norm of the estimated sources can be used as a contrast for the problem. We present a blind algorithm for the source separation of independents sources or mixtures of correlated sources by only a rotation matrix. We also present a variety of simulations assessing the performance of the proposed approach.
\end{abstract}

Index Terms-Bounded Component Analysis, Givens Rotations, Infinity Norm, Blind Source Separation.

\section{INTRODUCTION}

The problem of Blind Source Separation (BSS) [1], [2] consists in recovering a set of signals, called sources, which have been combined by a mixing system. In the case of linear and instantaneous mixing, with determined mixtures (number of mixtures equal to the number of sources), the mixtures $\mathbf{X}$ are given by:

$$
\mathbf{X}=\mathbf{H S}
$$

where $\mathbf{S}=\left[\begin{array}{llll}\mathbf{s}_{1} & \mathbf{s}_{2} & \cdots & \mathbf{s}_{N}\end{array}\right], \mathbf{s}_{i}=\left[s_{i}(0) \cdots s_{i}(T-\right.$ $1)]^{T}, \forall i=1,2, \cdots, N$, is the matrix containing all the $T$ available samples of the $N$ sources; $\mathbf{X}$ is defined in the same manner as $\mathbf{S}$; and $\mathbf{H} \in \mathbb{R}^{\mathrm{N} \times \mathrm{N}}$ is the mixing matrix.

For statistical independent signals, source separation is usually performed in two steps: a whitening preprocessing, multiplying the mixtures $\mathbf{X}$ with a whitening matrix, followed by a single rotation matrix.

The Independent Component Analysis (ICA) related techniques, usually find the separation matrix with the optimization of a statistical independence related criteria, such as mutual information, high-order statistics, nonlinear decorrelation and information theoretic learning criteria [3], [2]. In the last two decades several approaches

Paper submitted at September, $15^{\text {th }}$ 2021. The authors would like to thank FAPESP (processes number 2017/13025-2 and 2019/20899-4) and CAPES, Code 001.

Renan D. B. Brotto and João M. T. Romano are with the School of Electrical and Computer Engineering, University of Campinas, Campinas, São Paulo, Brazil (e-mail: rbrotto@decom.fee.unicamp.br, romano@decom.fee.unicamp.br).

Kenji Nose-Filho is with Center of Engineering, Modeling and Applied Social Sciences, Federal University of ABC (UFABC), Santo André, São Paulo, Brazil (e-mail: kenji.nose@ufabc.edu.br). were presented for the BSS problem, exploring alternative priors, such as source sparsity in Sparse Component Analysis (SCA) [4], [5], source disjointness in Disjoint Component Analysis (DCA) [6], [7], and the magnitude boundness of sources in Bounded Component Analysis (BCA) [8], [9], [10].

In this work, we present a new criteria for BCA, based on the sum of the $\ell_{\infty}$-norm of the estimated sources and show that it can be seen as a contrast function for bounded sources; we organized the paper as follows: in Section II we formalize the definition of bounded sources and present a theorem stating the sufficient conditions to recover them. In Section III, we present the $\ell_{\infty}$ norm as a contrast function alongside a suitable algorithm to recover bounded sources; from the theoretical results and the proposed algorithm, we performed numerical simulations and we present the results in Section IV. Finally, in Section V we state out the main conclusions of this work and its perspectives.

\section{THEORETICAL BACKGROUND}

The BCA criterion assumes that the sources present a limited amplitude range, alongside the condition that in some time instants all of them assume their maximum magnitude values. We formalize the notion of bounded sources in Definition 1.

Definition 1: We define a set of $N$ bounded sources, $\mathbf{s}(n)=\left[\begin{array}{llll}s_{1}(n) & s_{2}(n) & \cdots & s_{N}(n)\end{array}\right]^{T}$, as a set composed by signals with finite amplitude, $s_{i}(n) \in[-A, A], \forall i, n$, and for which all the sources assume all of the $2^{N}$ extreme values. For example, in the case of having two sources, there are $2^{4}$ time instants that the sources $\left\{s_{1}, s_{2}\right\}$ assume the values $\{A, A\},\{A,-A\},\{-A, A\}$, and $\{-A,-A\}$.

In other words, Definition 1 proposes that the sources are inside an hypercube with edges of length $2 A$, taking values in all of the $2^{N}$ vertices. Relying on the observation that the signals after the mixing process will no longer fit an hypercube, Theorem 1 suggests that, by fitting the data into an hypercube, it will recover the sources.

Theorem 1: Consider the set of $N$ bounded sources, $\mathbf{s}(n)=\left[\begin{array}{llll}s_{1}(n) & s_{2}(n) & \cdots & s_{N}(n)\end{array}\right]^{T}$, as proposed in Definition 1. Then, any linear combination $y(n)=$ 
$\sum_{l=1}^{N} g_{i, l} s_{l}(n)$, with $\left\|\mathbf{g}_{i}\right\|_{2}=1, \quad \forall i$, will have its infinity norm equalized, $\|y(n)\|_{\infty}=A$, if, and only if, $\mathbf{g}_{i}= \pm \delta_{i, j}$, for $j \in\{1,2, \ldots, N\}$.

Proof. Consider the estimate $y(n)=\sum_{l=1}^{N} g_{i, l} s_{l}(n)$ and its absolute value

$$
|y(n)|=\left|\sum_{l=1}^{N} g_{i, l} s_{l}(n)\right| .
$$

Using the fact that the absolute value of a sum is upper limited by the sum of its absolute values, we have

$$
\left|\sum_{l=1}^{N} g_{i, l} s_{l}(n)\right| \leq \sum_{l=1}^{N}\left|g_{i, l} s_{l}(n)\right| \leq A \sum_{l=1}^{N}\left|g_{i, l}\right| .
$$

where the second inequality holds from the finite support of the sources. Since there are time instants that allow the sources to assume their extreme values, we have

$$
\|y(n)\|_{\infty}=A \sum_{l=1}^{N}\left|g_{i, l}\right|=A\left\|\mathbf{g}_{i}\right\|_{1} .
$$

Since $\left\|\mathbf{g}_{i}\right\|_{2}=1$, from norm inequalities [11], it follows:

$$
\left\|\mathbf{g}_{i}\right\|_{1} \geq\left\|\mathbf{g}_{i}\right\|_{2}
$$

and we have the equality if, and only if, $g_{i}= \pm \delta_{i, j}$.

From (5), we have $\|y(n)\|_{\infty} \geq A$ and, once again, the equality occurs only for $g_{i}= \pm \delta_{i, j}$. So, the $\ell_{\infty}$ norm of the estimates will attain the minimum value only with separated sources, with a possible permutation and sign ambiguity.

Theorem 1 presents a very interesting result: if we have recovered the sources up to an orthogonal matrix, then the amplitude match is a sufficient condition to source separation, and the $\ell_{\infty}$ norm is a suitable contrast function. This case occurs when a) we have independent sources, pre-processed with a PCA-related technique. As the result, we have a set of decorrelated signals that corresponds to the sources, up to a rotation matrix; b) we have correlated sources, mixed by a rotation system.

In the next section we present an algorithm able to recover bounded sources for the two conditions presented above.

\section{RECOVERING BOUNDED SOURCES}

As stated in the previous section, Theorem 1 presents sufficient conditions to recover independent sources after a PCA step, or correlated ones, combined by a rotation system. Now, we are going to present a suitable algorithm to perform the separation.
From Theorem 1, the sum of the $\ell_{\infty}$-norm of the estimated sources, $\mathbf{Y}=\mathbf{W X}$, leads to the following inequality:

$$
J(\mathbf{W})=\sum_{i=1}^{N}\left\|\mathbf{y}_{i}\right\|_{\infty}=\sum_{i=1}^{N}\left\|\mathbf{w}_{i} \mathbf{X}\right\|_{\infty} \geq N A .
$$

The equality holds if, and only if, there is exactly one unitary element in each row and column of the global matrix $\mathbf{G}=\mathbf{W H}$, i.e., when the sources are separated.

Since $\mathbf{W}$ is an $N$-dimensional rotation matrix, we can decompose it as the multiplication of $N(N-1) / 2$ two-dimensional ones, one for each $\mathbf{s}_{i}-\mathbf{s}_{j}$ plane, $i=$ $1,2, \cdots N, j=i+1, \cdots N$. Such procedure can be achieved by means of the Givens rotations [12], [13] and is detailed in Algorithm 1.

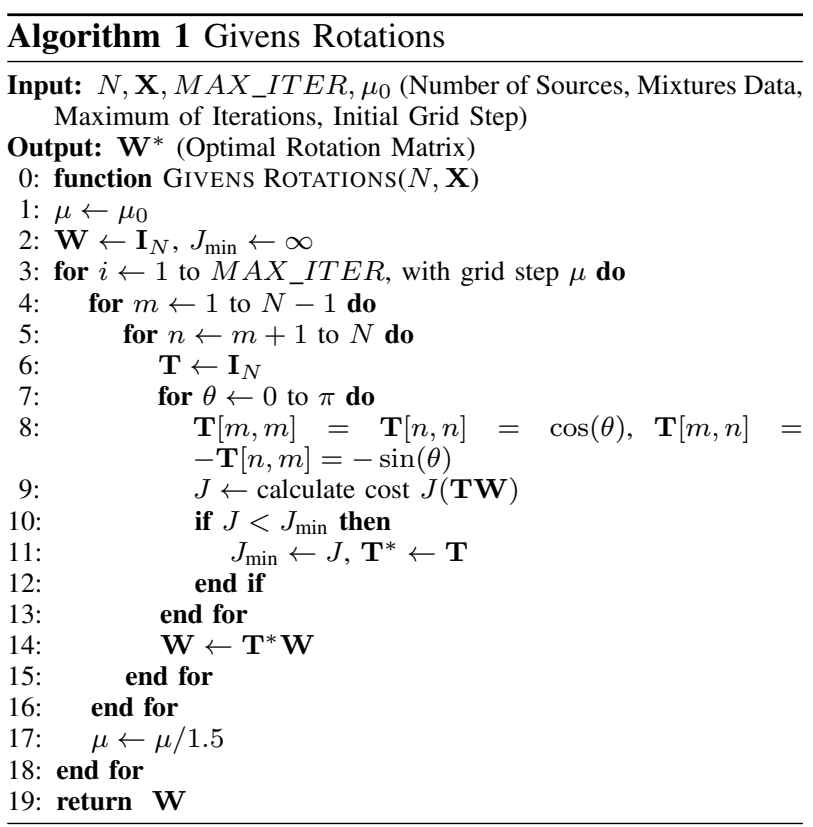

Since in Algorithm 1 we perform a grid search in a closed set, it will always converge to the minimum of (6). With the contrast function established and a suitable algorithm to optimize it, in the next section we present our simulation results.

\section{Simulation Results}

We applied Algorithm 1 in three classes of signals: Digital Communications, Gray Scale Images and Correlated Sources. In Table I we present all the performance metrics used in the numerical simulations.

\section{A. Digital Communication Signals}

We first considered a 4-PAM modulation scheme [14], with symbols $[ \pm 3, \pm 1], N=2,5,10$ sources, with $T=1000$ samples each. For the symbols distribution, we have considered a multi-modal one, where the symbols 


$$
\begin{aligned}
& \operatorname{SER}[\%]=\frac{\text { incorrect symbols }}{\text { transmitted symbols }} \times 100(7) \\
& \mathrm{Q}\left(\mathrm{s}_{i}\right)=\max _{j}\left(\frac{\sigma\left(s_{i}, \hat{s}_{j}\right)}{\sigma\left(s_{i}\right) \sigma\left(\hat{s}_{j}\right)} \cdot \frac{2 \overline{s_{i}} \overline{s_{j}}}{{\overline{i_{i}}}^{2}+\bar{s}_{j}{ }^{2}} \cdot \frac{2 \sigma\left(s_{i}\right) \sigma\left(\hat{s}_{j}\right)}{\sigma\left(s_{i}\right)^{2}+\sigma\left(\hat{s}_{j}\right)^{2}}\right) \text { (9) } \\
& \operatorname{ISI}(\mathrm{G})=\frac{1}{N} \sum_{i=1}^{N} \operatorname{ISI}\left(\mathbf{g}_{i}\right)(11) \\
& \begin{array}{r}
\operatorname{SIR}_{\mathrm{dB}}\left(\mathbf{s}_{i}\right)=\max _{j}\left(10 \log \left(\frac{\left\|\mathbf{s}_{i}\right\|^{2}}{\| \mathbf{s}_{i}-\left.\hat{\mathbf{s}}_{j}\right|^{2}}\right)\right)(8) \\
\operatorname{PSNR}\left(\mathrm{s}_{i}\right)=\max _{j}\left(10 . \log \left(\frac{\max _{n} s_{i}(n)^{2}}{\mathbb{E}\left[\left(s_{i}(n)-\hat{s}_{j}(n)\right)^{2}\right]}\right)\right)(10) \\
\operatorname{ISI}\left(\mathrm{g}_{i}\right)=10 \log _{10} \frac{\sum_{l}\left|g_{i, l}\right|^{2}-\max _{l}\left|g_{i, l}\right|^{2}}{\max _{l}\left|g_{i, l}\right|^{2}}(12)
\end{array}
\end{aligned}
$$

TABLE I: Performance Metrics.

\pm 3 present each a $37.5 \%$ of chance to occur (the other symbols present, naturally, a chance of $12.5 \%$ each).

We used Gaussian mixers, i.e., systems whose coefficients were drawn from a $\mathrm{N}(0,1)$ distribution. To better simulate real world scenarios, we added Gaussian noise to the mixtures, with 3 levels of SNR (Signal-to-Noise Ratio): $30 \mathrm{~dB}, 20 \mathrm{~dB}$ and $10 \mathrm{~dB}$. We compared our approach (Givens Rotations - GR) to another method designed for BCA the Ellipsoid Volume Maximization (VM), presented in [10], given by the cost function $J_{1}$. After the separation, we applied a decision rule, using the minimum Euclidean distance [14]. For the performance measure, we adopted the Symbol Error Rate (SER) (7) in 100 Monte Carlo simulations; we present the results obtained in Fig. 1.

We can observe that both methods presented a lower performance as we reduced the SNR or increased the number of sources, which was expected, since more noise and more signals render the problem more difficult. It is interesting to note that the proposed method presented a superior mean performance than the VM for SNRs of $30 \mathrm{~dB}$ and $20 \mathrm{~dB}$, but both methods attained almost the same SER level as we increase noise. Therefore, for lower or medium noise levels, the proposed method outperformed the comparative one, and got a closer performance to the VR as we increased the noise. This result is particularly interesting because it highlights the potential of BCA techniques to handle sources with multi-modal distribution, a great interest of the BSS field.

\section{B. Images}

We also applied the proposed method in the separation of gray scale images, comparing it again with [10]. We considered Gaussian mixers and the two independent images, as depicted in Fig. 2.

We evaluated both methods with three measures: SIR (Signal-to-Interference Ratio) (8) between the estimates $\hat{s}_{j}(n)$ and sources $s_{i}(n)$; the Image Quality Index (Q Index) (9) [15], where $\sigma(\cdot, \cdot)$ is the correlation coefficient, $\sigma(\cdot)$ the standard deviation and $\overline{s_{i}}$ the mean value; and the Peak Signal-to-Noise Ratio (PNSR) (10) [16]. We present the mean of these three metrics, taken in 10 Monte Carlo simulations, in Table II.

\begin{tabular}{|c|c|c|}
\hline Metric & GR & VM \\
\hline SIR $[d B]$ & $107.38 \pm 10.68$ & $107.29 \pm 30.55$ \\
\hline$Q$ Index $[0-1]$ & $1.00 \pm 10^{-4}$ & $1.00 \pm 10^{-4}$ \\
\hline$P S N R[d B]$ & $113.12 \pm 9.76$ & $113.03 \pm 30.37$ \\
\hline
\end{tabular}

TABLE II: Mean Performance of the 3 Metrics: SIR [dB], Q Index [0-1] and PSNR [dB].

It is interesting to note that the Q Index obtained by both methods reached the highest level, but it does not mean a complete image recovery, since this index takes only into account second-order statistics. On the other hand, the SIR and PSNR are measures that consider more structural characteristics of the sources and the higher they are, the better the estimates. From both the qualitative and quantitative measures, one can infer that the proposed method performs closely to the one presented in [10], with a smaller dispersion around the mean value. The proposed method, therefore, is suitable to recover gray scale images, a class of bounded signals with a broad range of applications.

\section{Correlated Sources}

Theorem 1 also states sufficient conditions to recover correlated sources, provided they were combined by a rotation system. To generate correlated sources, we first generated independent uniform ones, taking values in the interval $[-1,1]$, and then we applied the Copula-t technique, with 4 degrees of freedom, and a Toeplitz crosscorrelation matrix whose first row is $\left[\begin{array}{cccc}1 & \rho & \cdots & \rho^{N-1}\end{array}\right]$ [10], [17]. Once again, we performed the separation for $N=2,5$ and 10 sources, with $T=1000$ samples, varying the correlation index $\rho$ from 0.0 to 0.9 , and SNR of $15 \mathrm{~dB}$. To satisfy the conditions of Theorem 1 we added the extreme points $\{-1,1\}^{N}$.

We used the Intersymbol Interference (ISI) of the global matrix $\mathbf{G}$ as the performance measure (11) and (12) where $\mathbf{g}_{i}$ denotes the $i$-th row of $\mathbf{G}$. When one performs a perfect source separation, $\mathbf{g}_{i}=\delta_{i, j}$, and the $\operatorname{ISI}(\mathbf{G}) \rightarrow-\infty$; therefore, the lower the $\operatorname{ISI}(\mathbf{G})$, the better the source separation. We present our results, taken from 100 Monte Carlo Simulations, in Fig. 3.

For an SNR of $15 \mathrm{~dB}$, we notice that for 2 sources, both methods present a very close mean performance, and the VM approach is the less dispersed. For $N=$ 


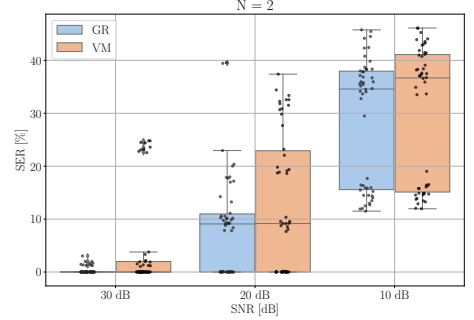

(a) 2 Sources

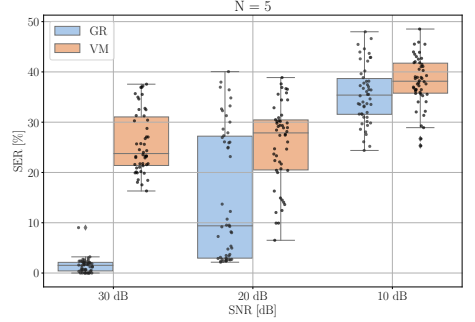

(b) 5 Sources

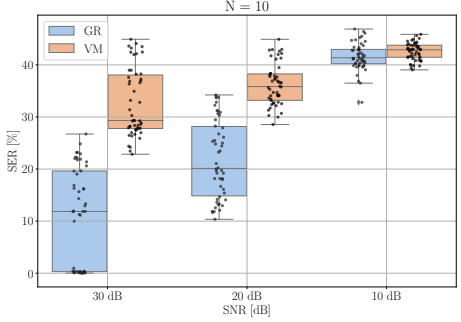

(c) 10 Sources

Fig. 1: Box plots and Swarmplots for SER Comparative.

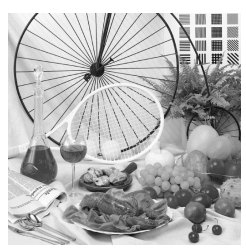

(a) Source 1 .

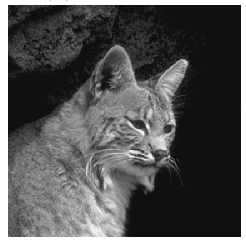

(e) Source 2.

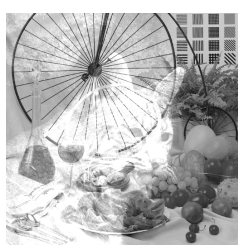

(b) Mixture 1.

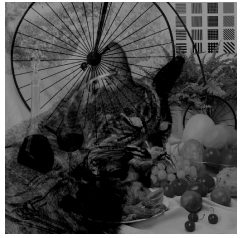

(f) Mixture 2.

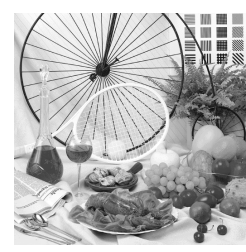

(c) Estimate 1 (GR).

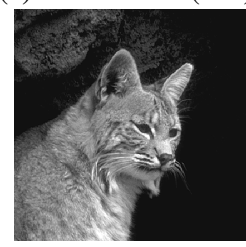

(g) Estimate 2 (GR).

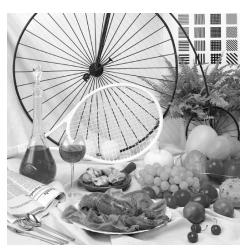

(d) Estimate 1 (VM).

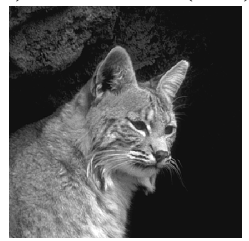

(h) Estimate 2 (VM).

Fig. 2: Performance Evaluation with Images.

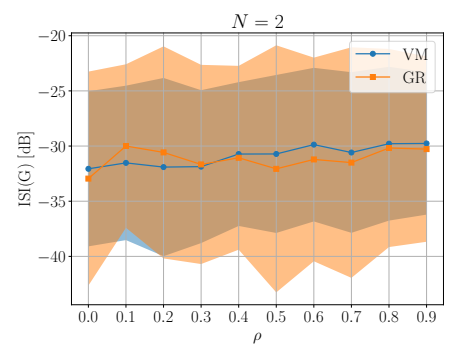

(a) 2 Sources

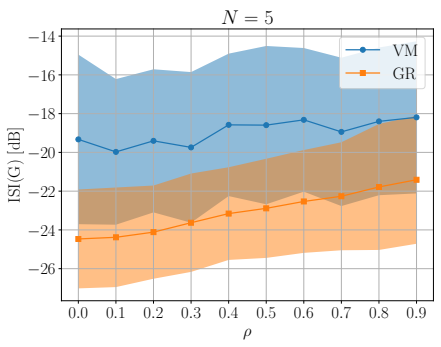

(b) 5 Sources

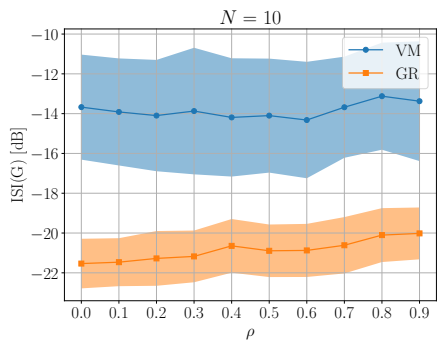

(c) 10 Sources

Fig. 3: Mean ISI (solid lines) and standard deviation (shadows) $\times \rho-\mathrm{SNR}=15 \mathrm{~dB}$.

5,10 , we observe again that the proposed method attained a better performance with less dispersion. Regarding the correlation level $\rho$, we note that this parameter hardly affects the ISI level obtained by 2 sources, but for $N=5,10$, we note lower performances for higher $\rho$. The performance robustness with respect to the correlation level occurs because the separation matrix structure, i.e., a rotation matrix, is not strongly affected by this parameter.

\section{CONCLUSION}

In this work we presented a BCA. We stated sufficient conditions to source recovery and we presented the $\ell_{\infty}$ norm as a contrast function for bounded signals. We evaluated the quality of our approach in a broad range of signals (digital communication, gray scale images and correlated sources with continuous amplitudes), considering different performance metrics. In all of the scenarios considered, the proposed method performed very well, with a performance as good as, or even better, than that obtained with the algorithm proposed in [10].

Since the proposed method led to a very interesting result for correlated sources, in future works we will investigate the development of a more general framework to handle dependent sources, considering general mixing systems. 


\section{REFERENCES}

[1] P. Comon and C. Jutten, eds., Handbook of blind source seapartion. Academic Press, 2010.

[2] J. M. T. Romano, R. Attux, C. C. Cavalcante, and R. Suyama, Unsupervised signal processing: channel equalization and source separation. CRC Press, 2011.

[3] A. Hyvarinen, J. Karhunen, and E. Oja, Independent component analysis. John Wiley \& Sons, 2001.

[4] R. Gribonval and S. Lesage, "A survey of sparse component analysis for blind source separation: principles, perspectives, and new challenges," in Proceedings of the 2006 ESANN, (Bruges, Belgium), pp. 323-330, 2006.

[5] L. T. Duarte, R. Suyama, R. Attux, J. M. T. Romano, and C. Jutten, "Blind extraction of sparse components based on $l_{0}$ norm minimization," in Proceedings of the 2011 SSP, pp. 617620, 2011.

[6] J. Anemuller, "Maximization of component disjointness: a criterion for blind source separation," in Independent Component Analysis, Springer Berlin Heidelberg, 2007.

[7] K. Nose-Filho, L. T. Duarte, and J. M. T. Romano, "On disjoint component analysis," in Latent Variable Analysis and Signal Separation (P. Tichavský, M. Babaie-Zadeh, O. J. Michel, and N. Thirion-Moreau, eds.), (Cham), pp. 519-528, Springer International Publishing, 2017.

[8] S. Cruces, "Bounded component analysis of linear mixtures: A criterion of minimum convex perimeter," IEEE Transactions on Signal Processing, vol. 58, no. 4, pp. 2141-2154, 2010.

[9] H. A. Inan and A. T. Erdogan, "An extended family of bounded component analysis algorithms," in 2014 48th Asilomar Conference on Signals, Systems and Computers, pp. 442-445, 2014.

[10] A. T. Erdogan, "A class of bounded component analysis algorithms for the separation of both independent and dependent sources," IEEE Transactions on Signal Processing, vol. 61, no. 22, pp. 5730-5743, 2013.

[11] S. Foucart and H. Rauhut, A Mathematical Introduction to Compressive Sensing. Springer, 1 ed., 2013.

[12] G. H. Golub and C. F. Van Loan, Matrix Computations. John Hopkins, 3rd ed., 1996.

[13] S. A. W. Shah, K. Abed-Meraim, and T. Y. Al-Naffouri, "Blind source separation algorithms using hyperbolic and givens rotations for high-order qam constellations," IEEE Transactions on Signal Processing, vol. 66, no. 7, pp. 1802-1816, 2018.

[14] J. Proakis and M. Salehi, Digital Communications. McGraw-Hill Education, 5th ed., 2007.

[15] Z. Wang and A. Bovik, "A universal image quality index," IEEE Signal Processing Letters, vol. 9, no. 3, pp. 81-84, 2002.

[16] Q. Huynh-Thu and M. Ghanbari, "Scope of validity of psnr in image/video quality assessment," Electronics Letters, vol. 44, no. 13, pp. 800-801, 2008.

[17] S. Demarta and A. J. McNeil, "The t copula and related copulas.," International Statistical Review / Revue Internationale De Statistique, vol. 73, no. 1, pp. 111-129, 2005. 